\title{
Should head and neck cancer survivors be considered a distinct special population within the context of exercise prescription?
}

Adrian W. Midgley ${ }^{1}$, Andrew R. Levy ${ }^{2}$, Ruth Price ${ }^{3}$, Felipe A. Cunha ${ }^{4}$, Simon N. Rogers ${ }^{5}$

1Prof Adrian W. Midgley, PhD, Department of Sport \& Physical Activity, Edge Hill University, Ormskirk, L39 4QP, UK. adrian.midgley@edgehill.ac.uk

${ }^{2}$ Dr Andrew R. Levy, PhD, Department of Psychology, Edge Hill University, Ormskirk, L39 4QP, UK. andy.levy@edgehill.ac.uk

${ }^{3}$ Ruth Price, Specialist Head and Neck Oncology Physiotherapist, University Hospital Aintree, Liverpool, L9 7AL, UK. RUTH.PRICE@aintree.nhs.uk

${ }^{4}$ Dr Felipe A. Cunha, PhD, Post-Graduate Program in Exercise Science and Sports, University of Rio de Janeiro State, Rio de Janeiro, Brazil, and Post-Graduate Program in Physical Activity Sciences, Salgado de Oliveira University, Niterói, Rio de Janeiro, Brazil. felipeac@globo.com

${ }^{5}$ Prof Simon N Rogers, FDS RCS FRCS MD, Faculty of Health and Social Care, Edge Hill University, Ormskirk, L39 4QP, UK and Consultant Regional Maxillofacial Unit, Aintree University Hospital, Liverpool, L9 1AE, UK. simonn.rogers@aintree.nhs.uk

ORCID. 0000-0002-5989-6142

\section{Corresponding author:}

Adrian W. Midgley

Department of Sport and Physical Activity

Edge Hill University

Ormskirk

L39 4QP

United Kingdom

Phone: 44 (0)1695 584318

Email: adrian.midgley@edgehill.ac.uk 


\begin{abstract}
Exercise is now widely accepted as a safe, cost-efficient, and effective intervention for cancer survivors and exercise guidelines for cancer survivors have been published. Currently there are no specific guidelines for different cancers despite apparent cancer-specific differences in the physical manifestations of the disease and the outcomes following treatment. One group of cancers where this appears to be particularly relevant is head and neck cancer (HANC). Common issues reported by HANC survivors include shoulder and neck dysfunction, head and neck lymphoedema, speech dysfunction, dysphagia, xerostomia, malnutrition, disfigurement, social isolation, and major depression. These issues have important implications for exercise prescription and supervision in terms of the safety and efficacy of the exercise, as well as patient uptake and adherence. HANC-specific issues should be considered when prescribing exercise within the framework of the general cancer exercise guidelines. Also, individual patient factors such as current fitness level, disease and treatment status, exercise preferences, and perceived barriers to exercise need to be considered. HANC survivors have many unique challenges and should be considered a distinct population within the context of exercise prescription. There has been a lack of research investigating exercise as an intervention in HANC survivors and much more research is required before robust evidence-based guidelines can be established. The purpose of this article is to provide a summary of salient factors to consider when optimising exercise prescription following HANC.
\end{abstract}

Key words: Fitness; oncology; physical activity; quality of life; training. 


\section{Introduction}

Exercise is now widely accepted as a safe, cost-efficient, and effective intervention for cancer survivors. ${ }^{1-}$ ${ }^{3}$ Considerable scientific evidence supports the efficacy of exercise for improving the quality of life of cancer survivors, ${ }^{3}$ helping prevent and manage co-morbidities, ${ }^{2}$ and reducing cancer-specific and allcause mortality. ${ }^{4}$ To inform medical and allied health professionals how best to prescribe this exercise, evidence-based guidelines have been published. ${ }^{5-9}$ An important issue, however, is currently there are no specific guidelines for different cancers despite apparent cancer-specific differences in the physical manifestations of the disease and its treatment. One group of cancers where this appears to be particularly relevant is head and neck cancer (HANC), which include malignancies on the lips, mouth, oropharynx, nasopharynx, larynx, salivary glands, and paranasal sinuses. ${ }^{10}$ Survivors of HANC share many of the same issues as other cancer cohorts such as intrusive treatments, dependency, the threat of recurrence, and early mortality. ${ }^{10}$ They also experience notable biopsychosocial issues either unique to HANC or more severe than in other cancers. ${ }^{11-12}$ The purpose of the present paper was to highlight how HANC survivors differ from survivors of other cancers, discuss the associated implications for prescribing safe and effective exercise, and consider whether HANC survivors should be regarded as a distinct special population within the context of exercise prescription.

\section{Characteristics}

Smoking, alcohol consumption, poor nutrition, and human papillomavirus are associated risk factors for HANC. ${ }^{13}$ More than two-thirds of HANC survivors are men $^{14}$ and typically come from low socioeconomic backgrounds, ${ }^{15}$ with a significant history of smoking and alcohol consumption. ${ }^{16}$ Pre-treatment physical activity levels and physical fitness are typically low, particularly in females, older individuals, and those with lower educational levels. ${ }^{17}$ Moreover, physical activity levels have been shown to be substantially reduced post-diagnosis and associated with low levels of physical fitness and functioning. ${ }^{18-19}$ Due to the cluster of lifestyle choices associated with poor health outcomes that are frequently observed in HANC survivor cohorts, comorbidities are common ${ }^{20}$ and are associated with further reductions in physical activity levels. ${ }^{17}$ 


\section{Physical issues}

Notably, HANC is regarded among the most debilitating of all cancers ${ }^{13}$ and HANC survivors are significantly frailer with more mobility impairments than individuals with other solid malignancies. ${ }^{21}$ Shoulder and neck dysfunction and pain resulting from radiation and surgical procedures used to treat HANC are common, with many of the treatment-related issues unique to this cancer cohort. ${ }^{22}$ Reported issues from neck dissection include loss of trapezius muscle strength, scapula winging, reduced active range of motion, and shoulder and neck pain. ${ }^{23}$ Exercise has the potential to improve function. McNeely et al., ${ }^{24}$ for example, observed that specific upper body progressive resistance exercises continued over 12-months significantly improved dissection-related functioning and quality of life. In the early posttreatment rehabilitation period shoulder and neck dysfunction will often be assessed and treated by a physical therapist. This is particularly important before a HANC survivor engages in a general exercise programme where there are significant problems associated with free flaps and accessory nerve dysfunction. Physical therapists will typically prescribe rehabilitation exercises to complete outside the clinic and those involved in general exercise prescription and supervision should be familiar with these exercises and possess at least a basic knowledge of any shoulder and neck dysfunction. This is to advise on correct technique and avoid exercises likely to exacerbate the problem. Shoulder and neck dysfunction and pain tend to cause pain-minimising postural deviations, ${ }^{25}$ which often promote abnormal movement patterns during exercise. ${ }^{8}$ This can exacerbate the current musculoskeletal issue and cause new issues further along the kinetic chain. ${ }^{26}$ It is therefore important to avoid or modify certain exercises and correct poor posture and movement compensations during exercise. Any chronic adaptive shortening and lengthening of skeletal muscles caused by cancer treatment also should be addressed with corrective exercise strategies. ${ }^{26}$

Secondary lymphoedema is a serious but often overlooked and underdiagnosed condition occurring in up to $75 \%$ of HANC survivors. ${ }^{27-28}$ It results from dysfunction of the cervicofacial lymphatic drainage caused by tumour invasion, surgical lymph node removal, and radiotherapy. ${ }^{27}$ Exercise was traditionally thought to be unsafe for those experiencing lymphoedema, although current evidence suggests that 
exercise prescribed according to current guidelines for cancer survivors has no effect on lymphoedema or its symptoms. ${ }^{29}$ Almost all research has been conducted on breast cancer survivors, however, and to the best of our knowledge no research has investigated the acute and chronic effects of exercise on lymphoedema in HANC survivors. Further research is needed to establish whether exercise is beneficial or should be contraindicated in those experiencing head and neck lymphoedema, and the efficacy of wearing prescribed facial compression garments. Until evidence-based guidelines are made available, it is prudent for those supervising exercise to have undergone training in recognising suspected lymphoedema, so that appropriate referrals to a medical professional can be made to obtain clearance for exercise. Those supervising exercise programmes typically will have weekly contact with HANC survivors, which is a good opportunity to advocate any lymphoedema prevention measures that have prescribed.

Trismus and a reduction in the ability to swallow (dysphagia) and salivate (xerostomia) are issues associated with surgery and radiation to the head and neck that can persist long-term. ${ }^{12,30-31}$ These issues have the potential to affect acute exercise responses and adherence. Dry mouth associated with xerostomia is particularly problematic, as it has long-term effects on almost all HANC survivors, including a considerable negative impact on quality of life. ${ }^{30}$ Notably, HANC survivors ranked dry mouth or throat as first of over 30 different barriers to exercise. ${ }^{32-33}$ This issue clearly has major implications for exercise uptake and adherence and future research could investigate how it can be addressed, including the efficacy of established management strategies for dry mouth. ${ }^{34}$ Increased salivary viscosity has been shown immediately post-exercise in apparently healthy individuals, ${ }^{35}$ however, to the best of our knowledge no research has investigated salivary viscosity and dry mouth symptoms in HANC survivors during and post-exercise. Exercise has the potential to exacerbate dry mouth symptoms in HANC survivors and it would be useful to investigate this issue to establish the extent of the problem. Dysphagia is a common symptom of HANC and can lead to chronic dehydration. ${ }^{36}$ Since the evaporation of sweat is the major mechanism of thermoregulation during exercise, ${ }^{37}$ fluid loss during exercise will 
exacerbate this issue. Consideration should therefore be given to the comfort and safety of exercise in HANC survivors prone to dehydration, especially if exercising in hot environments. ${ }^{38}$

Laryngectomy results in as significant compromise in the normal airway after HANC treatment. ${ }^{39}$ Breathing through a tracheostoma after total laryngectomy involves breathing environmental air that does not undergo the respiratory tract's natural humidifying and filtering functions. ${ }^{40}$ Exercising in cold and polluted air should therefore be avoided. Laryngectomy can result in the loss of voice ${ }^{41}$ and those supervising exercise should be mindful of communication difficulties, especially where these might impact on the safety of exercise. Swimming is effective for improving health ${ }^{42}$ and ranked the third most preferred mode of exercise in a survey of HANC survivors. ${ }^{33}$ Although swimming is generally regarded as safe, particularly because of the low impact and associated reduced musculoskeletal injury risk,$^{42}$ contact with water can be fatal in laryngectomised patients due to aspiration. ${ }^{43}$ Swimming is therefore contraindicated unless suitable aids such as the Larchel are used. ${ }^{43}$ Patients should be educated on this, along with clinical nurse specialist follow-up support to provide or signpost patients on to how to acquire such aids.

The incidence of malnutrition in HANC survivors before treatment can be as high as $63 \%$ and can rise to $88 \%$ during treatment. ${ }^{44}$ Critical weight loss is defined as the involuntary weight loss of $\geq 5 \%$ in one month or $\geq 10 \%$ in 6 months and research involving HANC survivors has reported a prevalence ranging from $31 \%$ to $57 \% .{ }^{45}$ Notably, $77.1 \%$ of weight loss has been shown to be attributable to loss of lean body mass. ${ }^{46}$ Exercise has the potential to promote weight loss by contributing to any negative energy balance that already exists, but conversely can promote weight gain by stimulating skeletal muscle hypertrophy. The nutritional status of the HANC survivor and the type and volume of exercise are therefore important considerations in attenuating weight loss and promoting muscle hypertrophy. Collaboration between an exercise profession and dietician is important to identify appropriate nutritional support for the intended level of physical activity and avoid exercise programmes that cannot be supported by adequate dietary intake. Enteral feeding tubes such as the percutaneous endoscopic gastronomy (PEG) tube are recommended for nutritionally vulnerable HANC survivors. ${ }^{47}$ In such cases, resistance exercises involving 
muscles in the area of the tube insertion and aquatic exercise or other microbial exposures should be avoided to help reduce the risk of dislodging the tube and infection, respectively. ${ }^{48}$

\section{Psychological and Emotional Issues}

Head and neck cancer is regarded as one of the most psychologically and emotionally traumatic cancer types. ${ }^{11}$ Depression has been reported to affect up to $50 \%$ of HANC survivors, with rates of major depressive disorder among the highest of all cancers. ${ }^{49}$ Patients with HANC are considered especially vulnerable to depression because of the frequent occurrence of physical and functional symptoms and mutilations after treatment. ${ }^{50}$ Exercise has been shown to be an effective intervention for reducing depression in people with different severities of depression, using a variety of exercise delivery strategies such as equipment-based or equipment-free modalities, inside or outside a hospital, indoors or outdoors, and alone or in groups. ${ }^{51}$ Notably, exercise interventions have compared favourably over antidepressant drugs and psychological interventions $\mathrm{s}^{51}$ and should therefore be promoted by the medical care team. Major depression is associated with poor exercise adherence, ${ }^{52}$ however, highlighting the need for behavioural change intervention $\mathrm{s}^{53}$ and additional support such as access to an emotional support therapist or psychologist. Exercise adherence in depressed individuals has been found to be higher when exercise programmes are delivered by appropriately trained exercise professionals. ${ }^{52}$ Although to the best of our knowledge this has not been established in HANC survivors, delivery of exercise programmes by exercise professionals is in accordance with the recommendations of the Clinical Oncology Society of Australia ${ }^{6}$ and seems a logical strategy.

Fatigue affects most HANC survivors to varying levels ${ }^{54}$ and was ranked sixth of 56 concerns that HANC survivors wished to discuss during their review consultation. ${ }^{55} \mathrm{~A}$ recent review of the literature found strong evidence for the efficacy of exercise interventions for decreasing cancer-related fatigue, with a dose-response relationship up to $150 \mathrm{~min} \cdot \mathrm{wk}^{-1}$ of aerobic exercise. ${ }^{8}$ Limited research has been conducted on the effects of exercise on fatigue in HANC survivors. Although it has been suggested that the research is generalisable across cancer types, ${ }^{8}$ more research specifically involving HANC survivors is required before more definitive guidelines can be provided. 
The highest prevalence of cancer-related pain of all cancers has been observed in HANC survivors, with $70 \%$ affected. ${ }^{56}$ Those supervising HANC survivors during exercise should be mindful of the implications of patients exercising while on pain medication. Pain medications dampen pain sensations during exercise, which increases the risk of tissue damage and injury. ${ }^{57}$ Ratings of perceived exertion is a recommended and well-established method of monitoring exercise intensity ${ }^{58}$ and pain medication has the potential to alter perceived exertion. Limited research suggests paracetamol does not alter perceived exertion during exercise, ${ }^{59}$ however, the effect of stronger analgesics has not been established. A second or alternative method of monitoring and regulating exercise, such as the percentage of heart rate reserve, may be prudent for those on strong pain medication to help avoid the potential for overexertion.

\section{Social Issues}

Visible disfigurement from HANC and its treatment has been shown to negatively impact on personal identity, ability to communicate with others, and the ability to feel successful interpersonally.$^{60}$ Those with disfigurement can react with social anxiety and behavioural avoidance in response to staring, comments, and unwanted questions ${ }^{61}$ and likely helps explain the high levels of social isolation observed in HANC survivors. ${ }^{62}$ Cancer rehabilitation classes exclusively for HANC survivors should prove useful in reducing social isolation and help address the lack of emotional support and social network that are major predictors of the depression observed in HANC survivors. ${ }^{50}$ The option of home exercise programmes is likely to be an important strategy in promoting exercise uptake and adherence in HANC survivors reluctant to exercise in social environments because of visible disfigurement. Since most HANC survivors are men, ${ }^{14}$ male dominance in group rehabilitation classes may be a barrier to women attending group classes ${ }^{63}$ and cancer rehabilitation should be tailored to meet the needs of women. Consequently, a menu-driven approach to exercise prescription offering different modes of delivery is likely the best strategy in promoting exercise uptake and adherence that better meets the needs of HANC survivors in regards the above social issues and substantial differences in their exercise preferences. $^{33}$ 


\section{Optimising Exercise Prescription}

Research investigating the influence of exercise on the risk of developing HANC is equivocal, ${ }^{16,64-66}$ however, current scientific evidence supports the efficacy of exercise for improving physical functioning and the quality of life of those already diagnosed with HANC. ${ }^{67}$ Exercise should therefore be promoted to all HANC survivors unless contraindicated. General physical activity guidelines for cancer survivors have been published ${ }^{5-9}$ and should be used as a framework for exercise prescription in HANC survivors. Current cancer physical activity guidelines recommend inclusion of exercises to develop cardiorespiratory fitness, muscle strength, and flexibility. ${ }^{5-9}$ Exercises to develop neuromotor fitness also may be considered to further promote a well-rounded exercise programme. Neuromotor exercises include those that develop balance, coordination, agility, and proprioception, and are regarded as particularly beneficial for older adults. ${ }^{58}$

Clearly, HANC survivors often have many cancer-specific issues that need to be considered when prescribing and supervising exercise and should therefore be considered a distinct population within the context of exercise prescription. Strategies to address HANC-specific issues should be applied within the context of an individualised approach to exercise prescription. This is in accordance with the recent criticism directed at the 'one size fits all' approach to prescribing exercise to cancer survivors that is likely hampering its full therapeutic effect. ${ }^{68-69}$ An individualised approach ideally should be guided by a comprehensive individual needs analysis that establishes the HANC survivor's current medical status, fatigue levels, musculoskeletal issues, lifestyle, exercise history, exercise preferences, perceived barriers to exercise, and goals, along with a carefully selected battery of fitness assessments. An established questionnaire such as the Patient Concerns Inventory for $\mathrm{HANC}^{55}$ also should prove helpful in individualising the exercise prescription to the patient's needs. The needs analysis and subsequent exercise prescription and supervision should ideally involve appropriately qualified exercise professionals. ${ }^{6,52}$ In the United Kingdom, for example, there are several accredited training providers of cancer rehabilitation exercise specialist qualifications for health and fitness professionals. The lack of availability of qualified cancer rehabilitation exercise specialists should not be perceived as a barrier to 
HANC survivors becoming generally more physically active, however. Education should ideally be provided to those prescribing and supervising exercise who are not specialists in HANC care, to help ensure HANC-specific issues are properly considered to optimise exercise safety and efficacy.

Optimising the content of an exercise programme is important for maximising its therapeutic effect, however, addressing exercise uptake and adherence is of primary importance, since a well-designed exercise programme will not be effective if not adhered to or taken up in the first place. Promoting exercise uptake and adherence in HANC survivors is likely to be particularly challenging due to the high prevalence of low socioeconomic backgrounds ${ }^{15}$, depression $^{49}$ and social isolation, ${ }^{62}$ which are all associated with poor exercise uptake and adherence. ${ }^{52,70-71}$ Another pertinent issue is that health interventions requiring voluntary uptake tend to increase health inequalities. ${ }^{72}$ Behavioural change strategies $^{73}$ are therefore particularly important in promoting exercise in HANC survivor cohorts. Despite the substantial evidence that 'exercise is medicine' for cancer survivors, a major criticism has been the lack of clinical staff that promote or prescribe exercise to their cancer patients. ${ }^{9}$ During the early stages of cancer rehabilitation, the exercise professional should be part of a multidisciplinary team, as is well established in cardiac rehabilitation. ${ }^{63}$ The role of clinical staff will be particularly important during the immediate post-diagnosis period in relation to discussing the benefits of exercise during cancer treatment and short and long-term recovery, as well as reassuring patients that exercise is safe. Exercise should be viewed as a key part of a HANC survivor's lifestyle and it is important that a consistent message is promoted by all members of the multidisciplinary team. Some HANC survivors may refuse to engage in exercises that promote specific components of fitness, such as strength training, or refuse to engage in any exercise altogether even after relevant education regarding its benefits. Personal choice regarding this should be respected. Important health benefits still can be gained from increasing nonexercise physical activity ${ }^{74}$ and the support team may need to be creative on how best to promote this in resistant individuals.

An important general principle is that cancer survivors should avoid being physically inactive ${ }^{8}$ and this point may be particularly pertinent to HANC survivors given the potential for high levels of physical 
deconditioning due to the very arduous treatment they often endure compared to other cancers. ${ }^{12}$ The exercise prescription itself and strategies to promote adherence will likely need to be adapted to account for biopsychosocial changes during the cancer journey and particularly during periods of treatment. Employing exercise as part of cancer prehabilitation is recommended to better prepare cancer survivors for the rigours of treatment, ${ }^{75}$ however, to the best of our knowledge the feasibility and efficacy of this strategy in HANC has not yet been established and research is required.

\section{Conclusion}

HANC survivors differ considerably from other cancers in ways that have important implications for exercise and should be regarded as a distinct special population within the context of exercise prescription. This differentiation is similar to many other diseases such as cardiovascular and pulmonary disease, where exercise prescription guidelines are provided for specific conditions within generic disease classifications. ${ }^{76-77}$ Exercise prescription in HANC has been severely under researched and much more is needed before robust evidence-based guidelines can be formulated. A major criticism is that exercise cancer research typically has not adhered to basic fitness training principles ${ }^{78}$ and future research investigating exercise in HANC survivors should endeavour to address this issue using contemporary and more individualised approaches to exercise prescription. 


\section{References}

1. Mewes JC, Steuten LMG, ljzerman MJ, et al. Effectiveness of multidimensional cancer survivor rehabilitation and cost-effectiveness of cancer rehabilitation in general: a systematic review. Oncologist 2012;17:1581-93.

2. Pederson BK, Saltin B. Exercise as medicine - evidence for prescribing exercise as therapy in 26 different chronic diseases. Scand J Med Sci Sports 2015;25 Suppl:1-72.

3. Gerritsen, JKW, Vincent AJPE. Exercise improves quality of life in patients with cancer: a systematic review and meta-analysis of randomised controlled trials. Br J Sports Med 2016;50:796-803.

4. McTiernan A, Friedenreich CM, Katzmarzyk PT, et al. Physical activity in cancer prevention and survival: a systematic review. Med Sci Sports Exerc 2019;51:1252-61.

5. Kushi LH, Doyle C, McCullough M, et al. American Cancer Society guidelines on nutrition and physical activity for cancer prevention: reducing the risk of cancer with healthy food choices and physical activity. CA Cancer J Clin 2012;62:30-67.

6. Cormie P, Atkinson M, Bucci L, et al. Clinical Oncology Society of Australia position statement on exercise in cancer care. Med J Aust 2018;209:184-7.

7. Hayes SC, Newton RU, Spence RR, et al. The Exercise and Sports Science Australia position statement: exercise medicine in cancer management. J Sci Med Sport 2019;22:1175-99.

8. Campbell KL, Winters-Stone KM, Wiskemann J, et al. Exercise guidelines for cancer survivors: consensus statement from international multidisciplinary roundtable. Med Sci Sports Exerc 2019;51:2375-90.

9. Schmitz KH, Campbell AM, Stuiver MM, et al. Exercise is medicine in oncology: engaging clinicians to help patients move through cancer. CA Cancer J Clin;2019;69:468-84.

10. Devins GM, Otto KJ, Irish JC, et al. Psychological issues related to site of cancer. In: Holland JC, Breitbart WS, Butow PN, et al. eds. Psycho-oncology. 3rd ed. New York (NY): Oxford University Press, 2015:92-7. 
11. De Boer MF, McCormick LK, Pruyn JF, et al. Physical and psychosocial correlates of head and neck cancer: a review of the literature. Otolaryngol Head Neck Surg 1999;120:427-36.

12. Sammut L, Ward M, Patel N. Physical activity and quality of life in head and neck cancer survivors: a literature review. Int J Sports Med 2014;35:794-9.

13. Taib BG, Oakley J, Dailey Y, et al. Socioeconomic deprivation and the burden of head and neck cancer regional variations of incidence and mortality in Merseyside and Cheshire, North West, England. Clin Otolaryngol 2018;43:846-53.

14. Lokker ME, Offerman MPJ, van der Velden L-A, et al. Symptoms of patients with incurable head and neck cancer: prevalence and impact on daily functioning. Head Neck 2013;35:868-76.

15. Conway DI, Brenner DR, McMahon AD, et al. Estimating and explaining the effect of education and income on head and neck cancer risk: INHANCE consortium pooled analysis of 31 case-controlled studies from 27 countries. Int J Cancer 2015;136:1125-39.

16. Hashibe M, Hunt J, Wei M, et al. Tobacco, alcohol, body mass index, physical activity, and the risk of head and neck cancer in the Prostrate, Lung, Colorectal, and Ovarian (PLCO) Cohort. Head Neck 2013;35:914-22.

17. Douma JAJ, Verdonck-de Leeuw IM, Leemans CR, et al. Demographic, clinical and lifestyle-related correlates of accelerometer assessed physical activity and fitness in newly diagnosed patients with head and neck cancer. Acta Oncol 2019; DOI: 10.1080/0284186X.2019.1675906.

18. Rogers LQ, Courneya KS, Robbins KT, et al. Physical activity and quality of life in head and neck cancer survivors. Support Care Cancer 2006;14:1012-9.

19. Gannon JA, Guinan EM, Doyle SL, et al. Reduced fitness and physic al functioning are long-term sequelae after curative treatment for esophageal cancer: a matched control study. Dis Esophagus 2017;30:1-7 
20. Eytan DF, Blackford AL, Eisele DW, et al. Prevalence of comorbidities and effect on survival in survivors of human papillomavirus-related and human papillomavirus-unrelated head and neck cancer in the United States. Cancer 2019;125:249-60.

21. Bras L, Driessen DAJJ, de Vries J, et al. Patients with head and neck cancer: are they frailer than patients with other solid malignancies? Eur J Cancer Care 2019;doi: 10.1111/ecc.13170.

22. Inoue H, Nibu K-I, Saito M, et al. Quality of life after neck dissection. Arch Otolaryngol Head Neck Surg 2006;132:662-6.

23. Gane EM, Michaleff ZA, Cottrell MA, et al. Prevalence, incidence, and risk factors for shoulder and neck dysfunction after neck dissection: a systematic review. Eur J Surg Oncol 2017;43:1199-218.

24. McNeely ML, Parliament MB, Seikaly H, et al. Sustainability of outcomes after a randomized crossover trial of resistance exercise for shoulder dysfunction in survivors of head and neck cancer. Physiother Can 2015;67:85-93.

25. Baggi F, Santoro L, Grosso E, et al. Motor and functional recovery after neck dissection: comparison of two early physical rehabilitation programmes. Acta Otorhinolaryngol Ital 2014;34:230-40.

26. Clark MA. An evidence-based approach to understanding human movement impairments. In: Clark MA, Lucett SC, Sutton BG. NASM essentials of corrective exercise training. Burlington (MA): Jones \& Bartlett Learning, 2014.

27. Deng J, Ridner SH, Murphy BA. Lymphedema in patients with head and neck cancer. Oncolol Nurs Forum 2011;38:E1-10.

28. Sember A, Pranskevich C, Scott ST, et al. Prehabilitation for lymphedema in head and neck cancer patients at a community cancer center. J Community Supportive Oncol 2017;15:e127-34.

29. Singh B, Disipio T, Peake J, et al. Systematic review and meta-analysis of the effects of exercise for those with cancer-related lymphedema. Arch Phys Med Rehabil 2016;97:302-15. 
30. Dirix P, Nuyts S, Poorten VV, et al. The influence of xerostomia after radiation therapy on quality of life: results of a questionnaire in head and neck cancer. Support Care Cancer 2008;16:171-9.

31. Weber C, Dommerich S, Pau HW, et al. Limited mouth opening after primary therapy of head and neck cancer. Oral Maxillofac Surg 2010;14:169-73.

32. Rogers LQ, Courneya KS, Robbins KT, et al. Physical activity correlates and barriers in head and neck cancer patients. Support Care Cancer. 2008;16:19-27.

33. Midgley AW, Lowe D, Levy AR, et al. Exercise program design considerations for head and neck cancer survivors. Eur Arch Otorhinolaryngol 2018;275:169-79.

34. AAOM. AAOM clinical practice statement: Subject: Clinical management of cancer therapy-induced salivary gland hypofunction and xerostomia. Oral Surg Oral Pathol Oral Radiol 2016;122:310-2.

35. Ligtenberg AJM, Liem EHS, Brand HS, et al. The effect of exercise on saliva viscosity. Diagnostics 2016;6:40.

36. Nund RL, Ward EC. Scarinci NA, et al. The lived experience of dysphagia following non-surgical treatment for head and neck cancer. Int J Speech Lang Pathol 2014;16:282-9.

37. Lim CL, Byrne C, Lee JKW. Human thermoregulation and measurement of body temperature in exercise and clinical settings. Ann Acad Med Singapore 2008;37:347-53.

38. Wendt D, van Loon, LC, van Marken Lichtenbelt WD. Thermoregulation during exercise in the heat: strategies for maintaining health and performance. Sports Med 2007;37:669-82.

39. Hutcheson KA, Alvarez CP, Barringer DA, et al. Outcomes of elective total laryngectomy for laryngopharyngeal dysfunction in disease-free head and neck cancer survivors. Otolaryngol Head Neck Surg 2012;146:585-90.

40. Hess MM, Schwenk RA, Frank W, et al. Pulmonary function after total laryngectomy. Laryngoscope 1999;109:988-94. 
41. Kapila M, Deore N, Palav RS, et al. A brief review of voice restoration following total laryngectomy. Indian J Cancer 2011;48:99-104.

42. Tanaka H. Swimming exercise: impact of aquatic exercise on cardiovascular exercise. Sport Med 2009;39:377-87.

43. Crevenna R, Schneider B, Mittermaier C, et al. Implementation of the Vienna Hydrotherapy Group for Laryngectomees - a pilot study. Support Care Cancer 2003;11:735-8.

44. Langius JAE, Bakker S, Rietveld DHF, et al. Critical weight loss is a major prognostic indicator for disease-specific survival in patients with head and neck cancer receiving radiotherapy. Br J Cancer 2013;109:1093-9.

45. Jager-Wittenaar H, Dijkstra PU, Vissink A, et al. Critical weight loss in head and neck cancerprevalence and risk factors at diagnosis: an explorative study. Support Care Cancer 2007;15:1045-50.

46. Silva HJ, Dietrich MS, Murphy BA. Changes in body mass, energy balance, physical function, and inflammatory state in patients with locally advanced head and neck cancer treated with concurrent chemotherapy after low-dose induction chemotherapy. Head Neck 2007;29:893-900.

47. Talwar B, Donnelly R, Skelly R, et al. Nutritional management in head and neck cancer: United Kingdom national multidisciplinary guidelines. J Laryngol Otol 2016;130:S32-40.

48. Buffart LM, Galvão DA, Brug J, et al. Evidence-based physical activity guidelines for cancer survivors: current guidelines, knowledge gaps and future research directions. Cancer Treat Rev 2014;40:327-40.

49. Lydiatt WM, Moran J, Burke WJ. A review of depression in the head and neck cancer patient. Clin Adv Hematol Oncol 2009;7:397-403.

50. de Leeuw JR, de Graeff A, Ros WJ, et al. Prediction of depressive symptomatology after treatment of head and neck cancer: the influence of pre-treatment physical and depressive symptoms, coping, and social support. Head Neck 2000;22:799-807. 
51. Morres ID, Hatzigeorgiadis A, Stathi A, et al. Aerobic exercise for adult patients with major depressive disorder in mental health services: a systematic review and meta-analysis. Depress Anxiety 2019;36:39-53.

52. Stubbs B, Vancampfort D, Rosenbaum S, et al. Dropout from exercise randomized controlled trials among people with depression: a meta-analysis and meta regression. J Affect Disord 2016;190:457-

66.

53. Perna FM, Craft L, Freund KM, et al. The effect of a cognitive behavioral exercise intervention on clinical depression in a multi-ethnic sample of women with breast cancer: a randomized controlled trial. Int J Sport Exerc Psychol 2010;8:36-47.

54. Bossi P, Di Pede P, Guglielmo M, et al. Prevalence of fatigue in head and neck cancer survivors. Ann Otol Rhinol Laryngol 2019;128:413-9.

55. Rogers SN, Thomson F, Lowe D. The Patient Concerns Inventory integrated as part of routine head and neck cancer follow-up consultations: frequency, case-mix, and items initiated by the patient. Ann R Coll Surg Engl 2018;100:209-15.

56. van dan Beuken-van Everdingen MHJ, de Rijke JM, Kessels AG, et al. Prevalence of pain in patients with cancer: a systematic review of the past 40 years. Ann Oncol 2007;18:1437-48.

57. Lundberg TR and Howatson G. Analgesic and anti-inflammatory drugs in sports: implications for exercise performance and training implications. Scand J Med Sci Sports 2018;28:2252-62.

58. Riebe D, Ehrman JK, Liguori G, et al. ACSM's guidelines for exercise testing and prescription. 10 th ed. Philadelphia (PA): Lippincott Williams \& Wilkins, 2018.

59. Esh CJ, Mauger AR, Palfreeman RA, et al. Acetaminophen (paracetamol): use beyond pain management and dose variability. Front Physiol 2017;8:1092.

60. Katz MR, Irish, JC, Devins GM, et al. Reliability and validity of an observer-rated disfigurement scale for head and neck cancer patients. Head Neck 2000;22:132-41. 
61. Rumsey N, Clarke A, White P, et al. Altered body image: appearance-related concerns of people with visible disfigurement. J Adv Nurs 2004;48:443-53.

62. Carlson RH. Head and neck cancer patients face social isolation, financial burdens. Oncology Times 2016;38(8):25.

63. Doherty P, Petre C, Onion N, et al. The national audit of cardiac rehabilitation: quality and outcomes report 2018. British Heart Foundation. Available from URL:

https://www.bhf.org.uk/informationsupport/publications/statistics/national-audit-of-cardiacrehabilitation-quality-and-outcomes-report-2018. (Last accessed 13 December 2019).

64. Nicolotti N, Chuang S-C, Cadoni G, et al. Recreational physical activity and risk of head and neck cancer: a pooled analysis within the international head and neck cancer epidemiology (INHANCE) Consortium. Eur J Epidemiol 2011;26:619-28.

65. Leitzmann MF, Koebnick C, Freedman ND, et al. Physical activity and head and neck cancer risk. Cancer Causes Control 2008;19:1391-9.

66. Lin C-L, Lee $\mathrm{W}-\mathrm{T}$, Ou C-Y, et al. Regular recreational physical activity and risk of head and neck cancer. BMC Cancer 2017;17:286.

67. Capozzi LC, Nishimura KC, McNeely ML. The impact of physical activity on health-related fitness and quality of life for patients with head and neck cancer: a systematic review. Br J Sports Med 2016;50:325-38

68. Kirkham AA, Bonsignore A, Bland KA, et al. Exercise prescription and adherence for breast cancer: one size does not FITT all. Med Sci Sports Exerc 2018;50:177-86

69. Brown JM, Shackelford DYK, Hipp ML, et al. Evaluation of an exercise-based phase program as part of a standard care module for cancer survivors. Translational J ACSM 2019;4:45-54.

70. Legh-Jones H, Moore S. Network social capital, social participation, and physical inactivity in an urban adult population. Soc Sci Med 2012;74:1362-7. 
71. Forechi L, Mill JG, Griep RH, et al. Adherence to physical activity in adults with chronic diseases: ELSA-Brasil. Rev Saude Publica 2018;52:31.

72. Macintyre S. Inequalities in Scotland: what are they and what can we do about them? Occasional paper No. 17. Glasgow: MRC Social \& Public Health Sciences Unit; 2007. Available from URL: file:///C:/Users/midglead/Downloads/OP017\%20(1).pdf. (Last accessed 13 December 2019).

73. Demark-Wahnefried W, Rogers LQ, Alfano CM, et al. Practical clinical interventions for diet, physical activity, and weight control in cancer survivors. CA Cancer J Clin 2015;65:167-89.

74. Chief Medical Officer. UK Chief Medical Officers' Physical Activity Guidelines, 2019. Available from URL:

https://assets.publishing.service.gov.uk/government/uploads/system/uploads/attachment data/file 1832868/uk-chief-medical-officers-physical-activity-guidelines.pdf. (Last accessed 13 December 2019).

75. Macmillan Cancer Support. Prehabilitation for people with cancer: principles and guidance for prehabilitation within the management and support of people with cancer; 2019. Available from URL: https://www.macmillan.org.uk/assets/prehabilitation-guidance-for-people-with-cancer.pdf. (Last accessed 13 December 2019).

76. Rundell KW, Smoliga JM, Weiss P. Pulmonary disorders and conditions. In: Jacobs PL, ed. NSCA's essentials of training specialist populations. Champaign (IL): Human Kinetics, 2018:145-79.

77. Swank AM, Sharp C. Cardiovascular conditions and disorders. In: Jacobs PL, ed. NSCA's essentials of training specialist populations. Champaign (IL): Human Kinetics, 2018:181-213.

78. Sasso JP, Eves ND, Christensen JF. A framework for exercise prescription in exercise-oncology research. J Cachexia Sarcopenia Muscle 2015;6:115-24.

\section{Conflict of interest}

We have no conflicts of interest. 